\title{
Análise da variabilidade da frequência cardíaca em indivíduos saudáveis, doentes com insuficiência cardíaca e doentes transplantados
}

\author{
Heart rate variability analysis in healthy subjects, patients suffering from \\ congestive heart failure and heart transplanted patients
}

\author{
A. Leite, M.E. Silva, A.P. Rocha
}

ARTIGO ORIGINAL | ORIGINAL ARTICLE

\begin{abstract}
O objetivo deste estudo foi determinar parâmetros de caracterização da variabilidade da frequência cardíaca (VFC) em indivíduos saudáveis e doentes que permitam a sua discriminação. Os parâmetros usados descrevem as diferentes componentes de memória (curta e longa) da VFC e são calculados por métodos paramétricos e não paramétricos. Assim, foram consideradas as componentes espectrais das baixas frequências (LF) e altas frequências (HF) associadas à memória curta e o parâmetro de memória longa $(d)$ obtidos por modelação autoregressiva de médias móveis integrados fracionariamente (ARFIMA). Analogamente, no contexto não paramétrico, foi utilizado o método detrended fluctuation analysis (DFA) no cálculo dos parâmetros de memória curta $\left(\alpha_{1}\right)$ e longa $\left(\alpha_{2}\right)$. A amostra foi constituída por 30 registos de 24 horas da base de dados Noltisalis: 10 indivíduos saudáveis, 10 doentes com insuficiência cardíaca e 10 doentes após transplante cardíaco. Observou-se que os parâmetros que caracterizam a memória curta apresentam valores mais elevados para o grupo dos indivíduos saudáveis, enquanto os parâmetros de memória longa têm valores mais elevados para os grupos dos doentes. Concluiu-se que a modelação ARFIMA permite discriminar os 3 grupos de dados em estudo, apresentando vantagens sobre o DFA.

Palavras-chave: análise espectral, memória longa, método DFA, modelos ARFIMA, variabilidade da frequência cardíaca
\end{abstract}

ABSTRACT

This study aimed to find parameters to characterize heart rate variability (HRV) and discriminate healthy subjects and patients with heart diseases. The parameters used for discrimination characterize the different components of HRV memory (short and long) and are extracted from HRV recordings using parametric as well as non parametric methods. Thus, the parameters are: spectral components at low frequencies (LH) and high frequencies (HF) which are associated with the short memory of HRV and the long memory parameter $(d)$ obtained from autoregressive fractionally integrated moving average (ARFIMA) models. In the non parametric context, short memory $\left(\alpha_{1}\right)$ and long memory $\left(\alpha_{2}\right)$ parameters are obtained from detrended fluctuation analysis (DFA). The sample used in this study contains 24-hour Holter HRV recordings of 30 subjects: 10 healthy individuals, 10 patients suffering from congestive heart failure and 10 heart transplanted patients from the Noltisalis database. It was found that short memory parameters present higher values for the healthy individuals whereas long memory parameters present higher values for the diseased individuals. Moreover, there is evidence that ARFIMA modeling allows the discrimination between the 3 groups under study, being advantageous over DFA.

Keywords: spectral analysis, long memory, detrended fluctuation analysis (DFA), ARFIMA models, heart rate variability

Submetido: 13.09.2012 | Aceite: 02.03.2013

Argentina Leite. Departamento de Matemática, Escola de Ciências e Tecnologia, Universidade de Trás-osMontes e Alto Douro e CM-UTAD, Vila Real, Portugal.

Maria Eduarda Silva. Faculdade de Economia, Universidade do Porto e CIDMA, Porto, Portugal.

Ana Paula Rocha. Departamento de Matemática, Faculdade de Ciências, Universidade do Porto e CMUP, Porto, Portugal.

Endereço para correspondência: Argentina Leite, Universidade de Trás-os-Montes e Alto Douro, Quinta de Prados, 5001-801 Vila Real, Portugal.

E-mail: tinucha@utad.pt 
Os sinais de natureza cardiovascular, como a frequência cardíaca, a pressão arterial e a forma dos complexos QRS no eletrocardiograma (ECG) apresentam variabilidade batimento a batimento. $\mathrm{O}$ estudo da variabilidade da frequência cardíaca (VFC) reveste-se de enorme importância, uma vez que permite aceder ao mecanismo de controlo do sistema cardiovascular, constituindo um meio não invasivo para estudar a sua integridade, em variados estados de doença (Apple, Berger, Saul, Smith, \& Cohen, 1989). O ECG é um dos meios de diagnóstico de mais baixo custo e de uso clínico mais generalizado. O tacograma, isto é, a sequência dos intervalos de tempo (RR) entre batimentos sucessivos no ECG é o sinal cardiovascular mais simples para a caracterização da VFC. A análise destes sinais e em particular a redução da VFC é suscetível de várias aplicações clínicas, em doenças cardiovasculares e não cardiovasculares (Task Force of European Society of Cardiology and the North American Society of Pacing Electrophysiology [ESC/NASPE], 1996).

A utilização das séries de VFC obtidas em regime ambulatório (registadores de Holter) é fundamental como meio auxiliar de diagnóstico e estratificação de risco. As séries de VFC de Holter são tipicamente longas, correspondendo a registos de 24 horas (aproximadamente 100000 batimentos) e permitem identificar patologias, nomeadamente arritmias malignas as quais não são detetadas nos registos de rotina do ECG. As séries longas de VFC apresentam características não estacionárias complexas, com ocorrência de pontos de mudança, de valores em falta ou outliers (batimentos incorretamente detetados) e evidenciando elevados e diferentes níveis de incerteza, decorrentes da variabilidade inter e intra individual e das diversas situações da vida diária.

O estudo dos sinais de VFC é efetuado recorrendo a métodos de processamento de sinal e análise de séries temporais no domínio do tempo e no da frequência (Task Force of ESC/NASPE, 1996). Para ilustrar a análise tradicional considere-se um tacograma de um indivíduo saudável, representado na Figura 1 (a): 512 batimentos no período do sono. O primeiro passo consiste em modelar o tacograma com um modelo autoregressivo (AR). Seguidamente faz-se uma análise espetral paramétrica AR do tacograma que está representada na Figura 1 (b). Esta análise permite identificar diversas componentes associadas à atividade do sistema nervoso autónomo (simpático e parassimpático), nomeadamente uma componente associada à atividade respiratória. No espectro é notória uma componente significativa abaixo de $0.04 \mathrm{~Hz}$, denominada de muito baixa frequência (VLF) e duas outras componentes nas baixas frequências (LF: 0.04$0.15 \mathrm{~Hz}$ ) e altas frequências (HF: 0.15-0.4 Hz), (a)

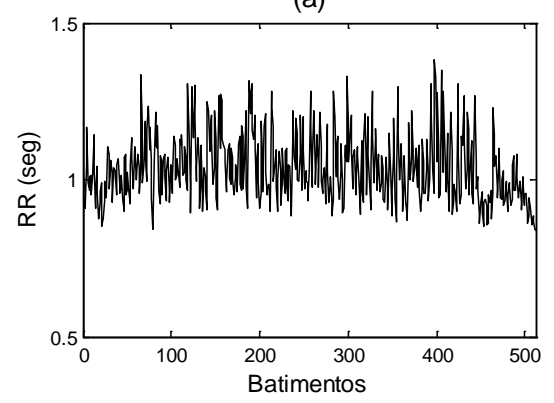

(b)

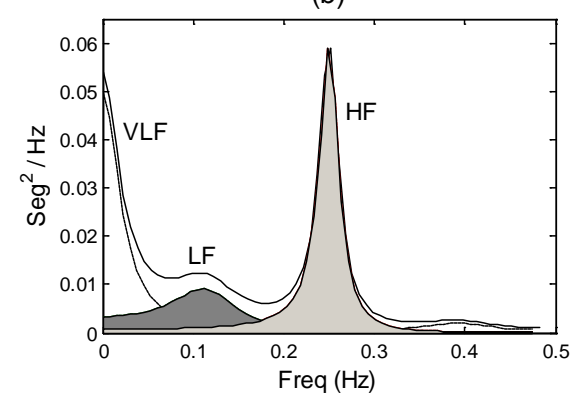

Figura 1. (a) Tacograma de um indivíduo saudável durante o período do sono (com 512 batimentos). Em (b) espectro paramétrico AR correspondente (obtido a partir das equações de Yule-Walker com resolução através do algoritmo de Durbin-Levinson) e decomposição automática do espectro nas componentes VLF, LF (marcada a cinza escuro) e HF (marcada a cinza claro) 
(a)

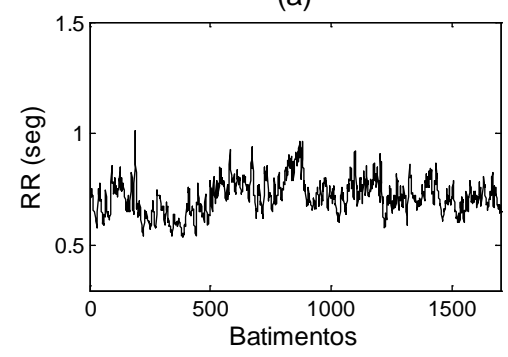

(b)
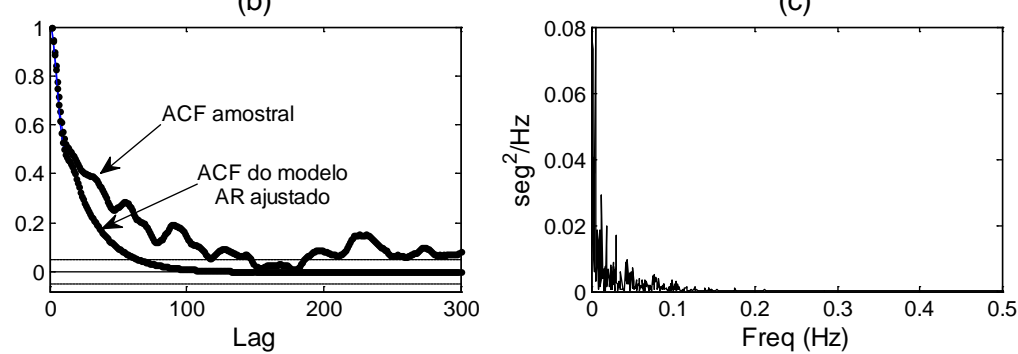

Figura 2. (a) Tacograma de um indivíduo saudável (com 1700 batimentos); (b) ACF amostral e ACF do modelo AR ajustado, com ordem 11 selecionada pelo critério de Akaike; (c) espectro correspondente. Bandas de confiança a $95 \%$ (--)

centradas respetivamente, na gama aproximada de $0.1 \mathrm{~Hz}$ e na frequência respiratória. É correntemente referido que a componente HF está relacionada apenas com a atividade do sistema nervoso parassimpático, enquanto a LF pode estar relacionada com a atividade do sistema nervoso simpático e parassimpático (Malik \& Camm, 1995). Alternativamente, poder-se-ia considerar uma análise espectral não paramétrica. No entanto, a análise paramétrica $A R$ apresenta, em relação a esta, a vantagem de permitir que as componentes espetrais sejam calculadas automaticamente através da identificação da frequência central de cada componente, como se ilustra na Figura 1 (b).

O tacograma pode ser adicionalmente caracterizado no domínio do tempo pela sua função de autocorrelação (ACF). A forma como se verifica o decaimento desta função pode interpretar-se como uma medida da memória do processo. As funções de autocorrelação amostral das séries de VFC apresentam um decaimento muito lento para zero, dito decaimento hiperbólico, designado na literatura por memória longa (Boukhan, Oppenheim, \& Taqqu, 2003). Esta característica indica que a dependência entre observações distantes apesar de ser pequena não é desprezável. No entanto, a ACF dos modelos AR apresenta uma característica diferente, decaindo exponencialmente para zero, sendo por isso os modelos AR designados por modelos de memória curta (Brockwell \& Davis, 1991). A característica de memória longa é ilustrada na Figura 2, considerando em (a) um tacograma de um indivíduo saudável e em (b) a correspondente ACF amostral e a ACF do modelo AR ajustado. Os registos curtos deste tipo são globalmente estacionários, apresentando, no entanto, ciclos ou tendências locais. No domínio da frequência, esta propriedade de memória longa reflete-se em espectros ilimitados próximo da origem, Figura 2 (c). Ao invés, a memória curta pode ser caracterizada na VFC através das componentes espectrais tradicionais, LH e HF.

A característica de memória longa em registos de VFC foi inicialmente observada por Kobayashi e Musha (1982) e posteriormente confirmada por Saul, Albrecht, Berger e Cohen (1987), usando um conjunto numeroso de registos de 24 horas. Esta característica tem sido usada na caracterização da VFC, permitindo detetar alterações nos registos de VFC mais eficientemente do que os parâmetros 
tradicionalmente usados (Mäkikallio, Perkiömäki, \& Huikuri, 2004). Em particular, permite distinguir entre indivíduos saudáveis e doentes (Bigger et al., 1996; Cerutti, Esposti, Ferrario, Sassi, \& Signorini, 2007; Peng, Havlin, Stanley, \& Golberger, 1995), caracterizar os períodos de dormir e acordado (Ivanov et al., 1999) e ainda monitorizar o efeito da idade (Iyengar, Peng, Morin, Goldberger, \& Lipsitz, 1996; Struzik, Hayano, Soma, Kwak, \& Yamamoto, 2006).

Para estimar a memória longa em registos não estacionários de VFC têm sido usados vários métodos não paramétricos, nomeadamente a rescaled range analysis (R/S) (Hurst, 1951), coarse graining spectral analysis (Yamamoto \& Hughson, 1991) e detrended fluctuation analysis (DFA) (Peng et al., 1995). O método não paramétrico DFA tem sido a técnica mais utilizada na estimação da componente de memória longa em registos de VFC (Baumert et al., 2012; Cerutti et al., 2007; Ivanov et al., 1999; Peng et al., 1995; Penzel, Kantelhardt, Grote, Peter, \& Bunde, 2003). Leite, Rocha, Silva e Costa (2006) propuseram em alternativa uma metodologia paramétrica, baseada nos modelos autoregressivos de médias móveis integrados fracionariamente (ARFIMA). Estes modelos são uma extensão dos modelos AR e incorporam a modelação conjunta de estruturas de correlação de termo curto e termo longo. Com efeito, a modelação ARFIMA permite estimar e remover a memória longa dos registos de VFC, conduzindo a uma melhor descrição dos parâmetros que caracterizam a memória curta, nomeadamente das componentes espectrais LF e HF (Leite et al., 2006).

Neste trabalho comparam-se os resultados da aplicação dos modelos AR, do método DFA e dos modelos ARFIMA em registos longos de 24 horas de VFC referentes a indivíduos saudáveis, doentes com insuficiência cardíaca e doentes após transplante cardíaco, com a finalidade de averiguar qual é a metodologia que permite discriminar os 3 grupos de dados em estudo.

\section{MÉTODO}

Trata-se de um estudo observacional, recorrendo a métodos de processamento de sinal e análise de séries temporais. Em particular, usam-se métodos tradicionais para a caracterização da VFC, tais como métodos no domínio do tempo e no da frequência (Task Force of ESC/NASPE, 1996). Consideram-se, ainda, métodos recentemente referidos na literatura (Leite et al., 2007) baseados em medidas não lineares, tais como DFA e ARFIMA. Opta-se por analisar dados previamente referidos na literatura, a base de dados Noltisalis (Signorini, Sassi, \& Cerutti, 2001), a fim de ser possível uma comparação de resultados.

Os registos constantes desta base de dados foram recolhidos através da colaboração entre Departamentos Universitários e clínicas de reabilitação em Itália, com a finalidade de estudar a natureza dos sinais de VFC a partir de uma perspetiva de séries temporais (Signorini et al., 2001).

Segundo os autores do estudo (Signorini, 2001) os dados relativos ao ECG foram registados em regime ambulatório com registadores de Holter de 24 horas. Seguidamente a extração das séries de VFC foi realizada com o auxílio de software adequado e validada por médicos.

\section{Amostra}

Neste trabalho analisou-se um conjunto de 30 registos de VFC referentes a três grupos de indivíduos da base de dados Noltisalis (Signorini et al., 2001): 10 registos de indivíduos saudáveis $(\mathrm{N}, 22.5 \pm 1.6$ horas; $102115.2 \pm$ 11365.4 batimentos; $42.2 \pm 6.4$ anos), 10 registos de indivíduos doentes com insuficiência cardíaca (C, $22.4 \pm 0.9$ horas; $107170.5 \pm$ 16689.3 batimentos; $53.6 \pm 11.2$ anos) e 10 registos de indivíduos doentes após transplante cardíaco (T, $22.4 \pm 0.7$ horas; 116043.3 \pm 11913.2 batimentos; $44.9 \pm 14.8$ anos).

A base de dados inclui informação acerca da hora de início de cada registo, bem como da idade de cada indivíduo. 


\section{Instrumentos e Procedimentos}

A descrição mais simples de registos longos e não estacionários de VFC pode ser obtida a partir de uma análise sequencial recorrendo a uma segmentação, isto é, decomposição do registo longo em segmentos curtos e aproximadamente estacionários. Os segmentos curtos são caracterizados por parâmetros obtidos pela aplicação das seguintes metodologias:

Domínio do tempo (Task Force of ESC/ NASPE, 1996): valor médio dos intervalos RR (meanRR) e a proporção de diferenças de intervalos $\mathrm{RR}$ sucessivos maiores que 50 msegundos (pNN50);

Modelação AR (Brockwell \& Davis, 1991): componentes espectrais LF e HF, designadas neste trabalho por $\mathrm{LF}_{\mathrm{AR}}$ e $\mathrm{HF}_{\mathrm{AR}}$, (estimadas a partir das equações de Yule-Walker com resolução através do algoritmo de Durbin-Levinson e com ordem do modelo otimizada pelo critério de informação de Akaike (AIC));

Método DFA (Peng et al., 1995): parâmetros de memória curta $\alpha_{1}$ (correspondente ao período de 4 a 11 batimentos; Pikkujämsä et al., 1999) e de memória longa $\alpha_{2}$ (correspondente ao período de 64 a 1024 batimentos; Leite et al., 2007);

Modelação ARFIMA (Leite et al., 2006): parâmetro de memória longa $d$ (obtido usando o estimador local de Whittle) e componentes espectrais $\mathrm{LF}_{\text {ARFIMA }}$ e $\mathrm{HF}_{\text {ARFIMA }}$ (estimadas após remoção da componente de memória longa).

A segmentação do registo longo em registos curtos pode ser fixa (todos ao segmentos têm a mesma dimensão fixa à partida) ou dita adaptativa se os segmentos têm dimensões variáveis dependendo das suas características. No cálculo dos parâmetros no domínio do tempo assim como na análise pelo método não paramétrico DFA, usam-se segmentos curtos de dimensão fixa (4096 batimentos, para permitir obter estimativas adequadas da memória longa; Leite et al., 2007). Já as metodologias paramétricas ARFIMA (ou AR), permitem que se considere uma segmentação adaptativa seletiva que é baseada no critério AIC. Neste caso, a dimensão dos segmentos curtos é variável e adaptada a cada registo (com dimensão mínima de 512 batimentos; Leite et al., 2006), permitindo obter uma descrição mais detalhada de períodos de transição (como o deitar e o levantar).

\section{Análise Estatística}

Com a finalidade de caracterizar os diferentes grupos de indivíduos em estudo, apresentam-se os resultados globais, considerando os valores médios dos vários parâmetros apresentados, calculados nos períodos de 24 horas e nos períodos de sono e acordado. A base de dados Noltisalis não dá indicação das horas referentes ao deitar e ao levantar, pelo que se consideram para o sono um período de 6 horas durante a noite e para o período acordado 6 horas durante o dia.

Com o objetivo de verificar se existem diferenças estatisticamente significativas entre os 3 grupos de dados aplicou-se inicialmente o teste de Shapiro-Wilk de normalidade. A utilização deste teste está relacionada com as dimensões reduzidas das amostras. Uma vez que as amostras se revelaram não normais optou-se pelo teste não paramétrico de Kruskal-Wallis com comparações múltiplas. No estudo considerouse um nível de significância de $5 \%$. Os métodos utilizados, bem como o tratamento estatístico dos dados foram realizados em ambiente Matlab.

\section{RESULTADOS E DISCUSSÃO}

Esta secção inicia-se com uma descrição detalhada da evolução ao longo do tempo dos parâmetros obtidos a partir da modelação ARFIMA para um registo típico de cada grupo. Com efeito, na Figura 3 representam-se registos de um indivíduo saudável, N6 (a), de um doente com insuficiência cardíaca, C10 (e) e de um doente após transplante cardíaco, T3 (i). Como seria de esperar, observa-se que a média dos intervalos RR apresenta valores mais elevados para os indivíduos saudáveis, assim como uma maior variabilidade. Relativamente aos valores do parâmetro de memória longa $d$, observa-se que variam ao longo do tempo, 
(a)

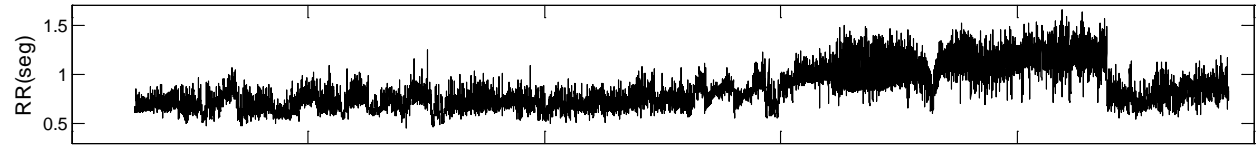

(b)

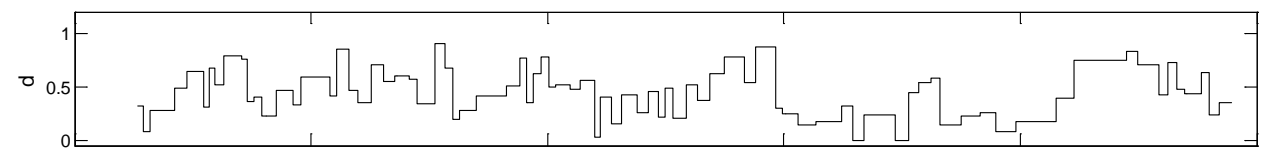

(c)

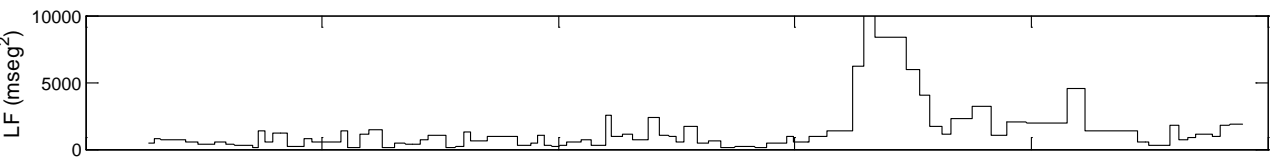

(d)

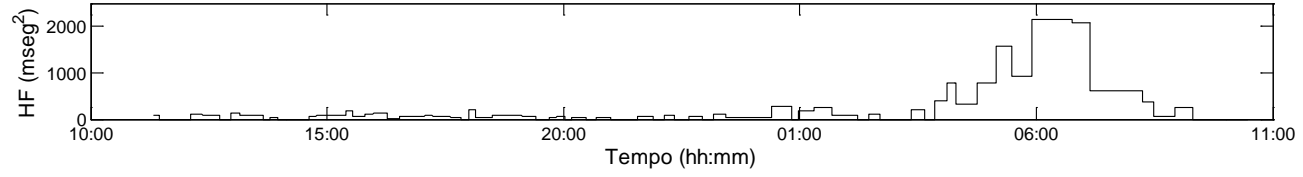

(e)

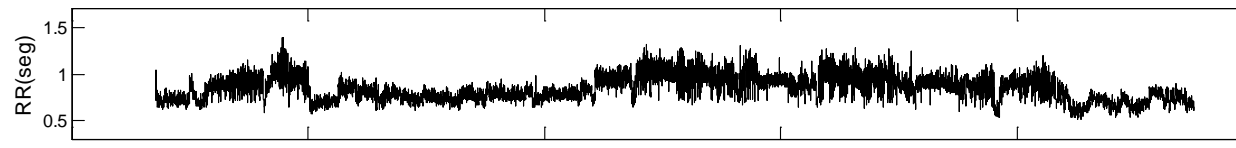

(f)

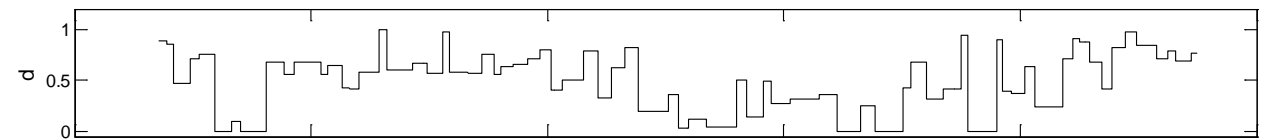

(g)

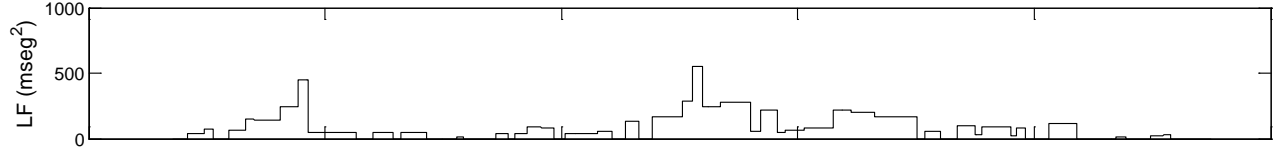

(h)

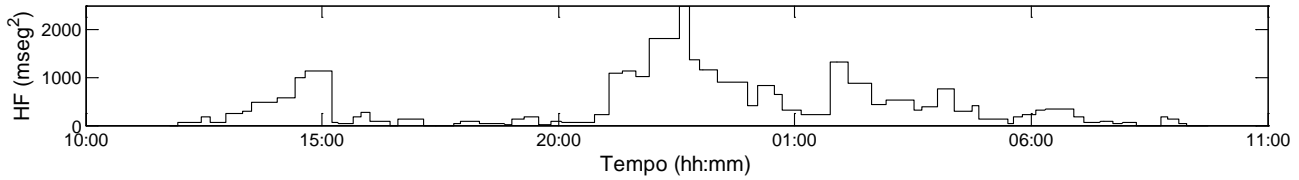

(i)

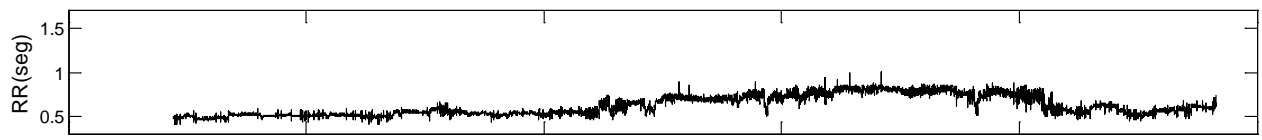

(j)

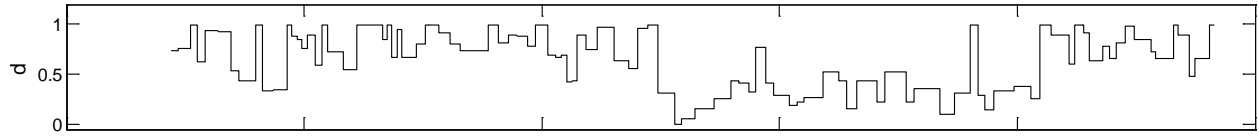

(k)

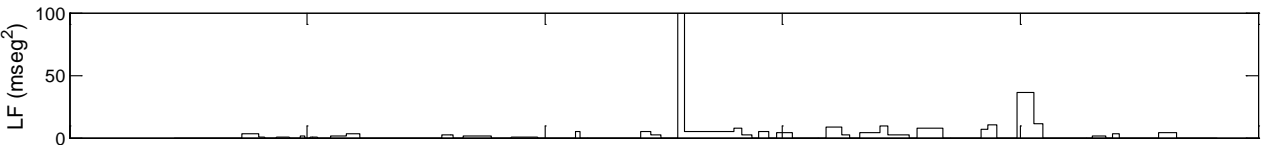

(l)

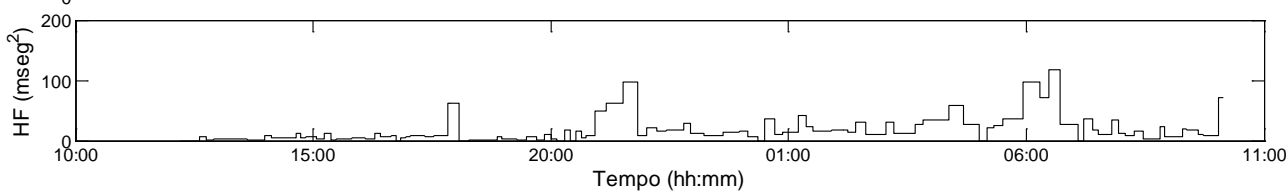

Figura 3. Tacogramas de três registos de Holter de 24 horas, referentes ao indivíduo saudável N6 (a), ao doente com insuficiência cardíaca C10 (e) e ao doente após transplante cardíaco T3 (i). Seguimento ao longo das 24 horas do parâmetro de memória longa $d$ e das componentes espetrais LF $_{\text {ARFIMA }}$ e $\mathrm{HF}_{\mathrm{ARFIMA}}$, obtidos usando a segmentação adaptativa seletiva combinada com modelação ARFIMA 
Tabela 1

Estimativas (média \pm desvio padrão) de vários parâmetros para os três grupos de dados da base de dados Noltisalis: indivíduos saudáveis $(N)$, doentes com insuficiência cardíaca $(C)$ e doentes após transplante cardíaco $(T)$, durante as 24 horas, 6 horas do período da noite e 6 horas do período de dia

\begin{tabular}{|c|c|c|c|c|c|}
\hline Método & Parâmetro & Período & $\mathrm{N}$ & $\mathrm{C}$ & $\mathrm{T}$ \\
\hline \multirow{6}{*}{$\begin{array}{c}\text { Domínio } \\
\text { do } \\
\text { Tempo }\end{array}$} & \multirow{3}{*}{ meanRR(seg) } & $24 \mathrm{~h}$ & $0.80 \pm 0.07$ & $0.77 \pm 0.11$ & $0.70 \pm 0.09$ \\
\hline & & Dormir $-6 h$ & $0.95 \pm 0.09$ & $0.86 \pm 0.12$ & $0.78 \pm 0.08$ \\
\hline & & Acordado $-6 \mathrm{~h}$ & $0.72 \pm 0.05$ & $0.72 \pm 0.10$ & $0.66 \pm 0.10$ \\
\hline & \multirow{3}{*}{ pNN50 (\%) } & $24 \mathrm{~h}$ & $4.4 \pm 3.1$ & $1.0 \pm 1.5$ & $0.4 \pm 0.7$ \\
\hline & & Dormir $-6 h$ & $7.0 \pm 6.7$ & $2.1 \pm 3.6$ & $0.5 \pm 0.6$ \\
\hline & & Acordado $-6 h$ & $4.2 \pm 4.8$ & $0.3 \pm 0.4$ & $0.3 \pm 0.5$ \\
\hline \multirow{7}{*}{$\begin{array}{c}\text { Modelação } \\
\text { AR }\end{array}$} & ordem & $24 \mathrm{~h}$ & $11.29 \pm 1.06$ & $11.70 \pm 1.98$ & $13.47 \pm 1.93$ \\
\hline & \multirow{3}{*}{$\mathrm{LF}_{\mathrm{AR}}\left(\mathrm{mseg}^{2}\right)$} & $24 \mathrm{~h}$ & $987 \pm 525$ & $74 \pm 78$ & $10 \pm 10$ \\
\hline & & Dormir $-6 h$ & $1148 \pm 761$ & $96 \pm 123$ & $11 \pm 10$ \\
\hline & & Acordado $-6 \mathrm{~h}$ & $1072 \pm 922$ & $44 \pm 35$ & $7 \pm 10$ \\
\hline & \multirow{3}{*}{$\mathrm{HF}_{\mathrm{AR}}\left(\mathrm{mseg}^{2}\right)$} & $24 \mathrm{~h}$ & $233 \pm 195$ & $84 \pm 86$ & $29 \pm 19$ \\
\hline & & Dormir - 6h & $384 \pm 312$ & $154 \pm 209$ & $43 \pm 37$ \\
\hline & & Acordado $-6 \mathrm{~h}$ & $217 \pm 324$ & $38 \pm 36$ & $20 \pm 19$ \\
\hline \multirow{6}{*}{$D F A$} & \multirow{3}{*}{$\alpha_{1}$} & $24 \mathrm{~h}$ & $1.47 \pm 0.18$ & $1.19 \pm 0.29$ & $0.71 \pm 0.24$ \\
\hline & & Dormir - $6 \mathrm{~h}$ & $1.35 \pm 0.15$ & $1.16 \pm 0.28$ & $0.77 \pm 0.28$ \\
\hline & & Acordado $-6 h$ & $1.53 \pm 0.26$ & $1.20 \pm 0.33$ & $0.67 \pm 0.28$ \\
\hline & \multirow{3}{*}{$\alpha_{2}$} & $24 \mathrm{~h}$ & $0.93 \pm 0.04$ & $1.02 \pm 0.11$ & $1.33 \pm 0.20$ \\
\hline & & Dormir - 6h & $0.78 \pm 0.07$ & $0.91 \pm 0.13$ & $1.24 \pm 0.24$ \\
\hline & & Acordado $-6 \mathrm{~h}$ & $0.97 \pm 0.04$ & $1.06 \pm 0.11$ & $1.36 \pm 0.22$ \\
\hline \multirow{10}{*}{$\begin{array}{c}\text { Modelação } \\
\text { ARFIMA }\end{array}$} & ordem & $24 \mathrm{~h}$ & $9.06 \pm 1.45$ & $9.25 \pm 2.21$ & $12.00 \pm 2.03$ \\
\hline & \multirow{3}{*}{$\mathrm{LF}_{\text {ARFIMA }}\left(\mathrm{mseg}^{2}\right)$} & $24 \mathrm{~h}$ & $688 \pm 403$ & $64 \pm 86$ & $6 \pm 5$ \\
\hline & & Dormir - 6h & $840 \pm 990$ & $86 \pm 128$ & $10 \pm 13$ \\
\hline & & Acordado $-6 \mathrm{~h}$ & $767 \pm 527$ & $37 \pm 40$ & $3 \pm 5$ \\
\hline & \multirow{3}{*}{$\mathrm{HF}_{\text {ARFIMA }}\left(\mathrm{mseg}^{2}\right)$} & $24 \mathrm{~h}$ & $292 \pm 254$ & $108 \pm 109$ & $39 \pm 34$ \\
\hline & & Dormir - 6h & $488 \pm 370$ & $189 \pm 239$ & $57 \pm 44$ \\
\hline & & Acordado $-6 h$ & $235 \pm 405$ & $60 \pm 53$ & $26 \pm 29$ \\
\hline & \multirow{3}{*}{$d$} & $24 \mathrm{~h}$ & $0.44 \pm 0.06$ & $0.52 \pm 0.14$ & $0.76 \pm 0.10$ \\
\hline & & Dormir $-6 h$ & $0.33 \pm 0.05$ & $0.38 \pm 0.16$ & $0.67 \pm 0.17$ \\
\hline & & Acordado $-6 h$ & $0.48 \pm 0.08$ & $0.59 \pm 0.16$ & $0.79 \pm 0.12$ \\
\hline
\end{tabular}

indicando que estes registos apresentam características de multifractalidade. Mais, os valores de $d$ apresentam uma variação circadiana com valores mais baixos durante o período do sono (de noite). Estes resultados estão de acordo com os obtidos por Leite et al. (2007), que aplicaram modelos ARFIMA em registos de 24 horas de VFC referentes a indivíduos saudáveis jovens e idosos, e com os obtidos por Baillie, Cecen, e Erkal (2009), que também usaram modelos ARFIMA num registo de 24 horas referente a um indivíduo saudável. No que respeita às componentes espetrais $\mathrm{LF}_{\mathrm{ARFIMA}} \mathrm{e}$ $\mathrm{HF}_{\mathrm{ARFIMA}}$, observa-se que também apresentam uma variação circadiana mas com valores mais elevados durante o período do sono. Estes 
resultados são consistentes com os obtidos por Leite et al. (2006) num registo de 24 horas de VFC referente a um indivíduo saudável.

Os resultados globais da aplicação das metodologias referidas na seç̧ão Instrumentos e Procedimentos estão sumariados na Tabela 1 para os 3 grupos de indivíduos. Observa-se que os valores médios dos parâmetros no domínio do tempo (meanRR e pNN50) e dos parâmetros que caracterizam a memória curta (componentes espetrais $\mathrm{LF}_{\mathrm{AR}}, \mathrm{HF}_{\mathrm{AR}}, \mathrm{LF}_{\mathrm{ARFIMA}}$ e $\mathrm{HF}_{\mathrm{AR}}$ FIMA $\mathrm{e} \alpha_{1}$ ) apresentam valores mais baixos para os grupos dos doentes do que para o grupo dos indivíduos saudáveis, quer durante o período de sono, quer no período de acordado. Em particular, os doentes transplantados apresentam valores mais baixos do que os doentes com insuficiência cardíaca. Verifica-se que os valores médios dos parâmetros de memória longa $\left(\alpha_{2}\right.$ e $\left.d\right)$ são mais elevados para os grupos dos doentes do que para o grupo dos indivíduos saudáveis, quer durante o período de sono, quer no período de acordado. Em particular, observa-se que os doentes após transplante cardíaco têm valores mais elevados do que os doentes com insuficiência cardíaca. Estes resultados são consistentes com os obtidos por Cerutti et al. (2007) os quais calcularam um único parâmetro de memória longa para cada registo de 24 horas da base de dados Nortisalis, considerando o método DFA. A metodologia ARFIMA descrita no presente trabalho tem a vantagem de permitir o seguimento ao longo do tempo da memória longa. Relativamente às ordens dos modelos AR e ARFIMA, verifica-se que estas apresentam valores médios mais elevados para os grupos dos indivíduos doentes. Além disso, observa-se que a ordem do modelo ARFIMA é tipicamente inferior à do modelo AR, o que está de acordo com Leite et al. (2006) que aplicam modelos AR e ARFIMA num registo de VFC referente a um indivíduo saudável. Quanto à dimensão dos segmentos curtos, observa-se que a metodologia ARFIMA permite obter segmentos curtos com dimensão inferior a 4096 batimentos (dimensão mínima para aplicar o método DFA). Com efeito, o valor médio da dimensão dos segmentos curtos aplicando modelos ARFIMA é 1287 batimentos para o grupo N, 1317 batimentos para o grupo C e 1328 batimentos para o grupo $\mathrm{T}$.

Finalmente, avaliaram-se as diferenças estatísticas entre os 3 grupos de indivíduos usando os dados relativos às 24 horas (período 24 horas) através do teste de Kruskal-Wallis com comparações múltiplas. Na Tabela 2 suma-

Tabela 2

Comparação dos três grupos de dados, durante as 24 horas, através do teste de Kruskal-Wallis com comparações múltiplas

\begin{tabular}{|c|c|c|c|c|c|}
\hline \multirow{2}{*}{ Método } & \multirow{2}{*}{ Parâmetro } & \multirow{2}{*}{$\begin{array}{l}\text { Teste de Kruskal-Wallis } \\
\text { p-value }\end{array}$} & \multicolumn{3}{|c|}{ Comparação Múltipla } \\
\hline & & & $\mathrm{N} v s . \mathrm{C}$ & $\mathrm{N} v s . \mathrm{T}$ & C vs.T \\
\hline \multirow{2}{*}{$\begin{array}{l}\text { Domínio } \\
\text { do Tempo }\end{array}$} & meanRR & .0969 & - & - & - \\
\hline & pNN50 & .0003 & $*$ & $*$ & - \\
\hline \multirow{2}{*}{$\begin{array}{c}\text { Modelação } \\
A R\end{array}$} & $\mathrm{LF}_{\mathrm{AR}}$ & $<.0001$ & * & * & - \\
\hline & $\mathrm{HF}_{\mathrm{AR}}$ & .0005 & - & $*$ & - \\
\hline \multirow{2}{*}{$D F A$} & $\alpha_{1}$ & $<.0001$ & - & $*$ & - \\
\hline & $\alpha_{2}$ & $<.0001$ & - & $*$ & $*$ \\
\hline \multirow{3}{*}{$\begin{array}{l}\text { Modelação } \\
\text { ARFIMA }\end{array}$} & $\mathrm{LF}_{\text {ARFIMA }}$ & $<.0001$ & * & * & - \\
\hline & $\mathrm{HF}_{\text {ARFIMA }}$ & .0005 & - & $*$ & - \\
\hline & $d$ & .0001 & - & $*$ & $*$ \\
\hline
\end{tabular}

Nota: Apresenta-se o nível de significância do teste e o símbolo * indica a presença de diferenças estatísticas entre os grupos 
riam-se os resultados. Verifica-se que os grupos $\mathrm{N}$ e $\mathrm{C}$, e $\mathrm{N}$ e $\mathrm{T}$ diferem relativamente ao parâmetro no domínio do tempo pNN50, ao nível de significância de 5\%. As componentes espectrais $L_{\mathrm{AR}}$ e $\mathrm{HF}_{\mathrm{AR}}$ obtidas pela modelação AR também permitem discriminar os mesmos grupos. Os parâmetros $\alpha_{1}$ e $\alpha_{2}$ do método DFA diferem entre $\mathrm{N}$ e $\mathrm{T}$ e entre $\mathrm{C}$ e $\mathrm{T}$. Os parâmetros obtidos a partir da modelação ARFIMA, parâmetro de memória longa $d$ e componentes espectrais $\mathrm{LF}_{\text {ARFIMA }}$ e $\mathrm{HF}_{\text {ARFIMA }}$, apresentam a vantagem de permitir discriminar entre os 3 grupos de dados. Conclusões análogas foram obtidas para os períodos dormir (6 horas) e acordado (6 horas).

\section{CONCLUSÕES}

A partir dos resultados encontrados e assumindo a limitação da especificidade da base de dados em estudo concluiu-se que os parâmetros correntemente utilizados para caracterizar a VFC não são suficientes para a discriminação completa dos 3 grupos de pacientes.

Com efeito, verificou-se que os parâmetros $\mathrm{LF}_{\mathrm{AR}}$ e $\mathrm{HF}_{\mathrm{AR}}$ obtidos a partir da modelação tradicional AR apenas permitem discriminar entre os grupos dos indivíduos saudáveis e dos doentes. Por outro lado, os parâmetros de memória curta, $\alpha_{1}$, e de memória longa, $\alpha_{2}$, obtidos pelo método não paramétrico DFA permitem discriminar entre os grupos normais e transplantados e entre os grupos de pacientes com insuficiência cardíaca e transplantados.

$O$ presente estudo revelou ainda que os parâmetros obtidos pela modelação ARFIMA, parâmetros LF $_{\text {ARFIMA }}$ e $\mathrm{HF}_{\text {ARFIMA }}$ associados à memória curta e o parâmetro de memória longa, $d$, apresentam a vantagem de permitirem discriminar os 3 grupos de pacientes, com potencial impacto na prática e no auxílio ao diagnóstico clínico.

Agradecimentos:

Os autores agradecem a disponibilização da base de dados Noltisalis (Signorini et al., 2001).
Conflito de Interesses:

Nada a declarar.

\section{Financiamento:}

Este trabalho foi financiado por Fundos Nacionais através da FCT - Fundação para a Ciência e a Tecnologia no âmbito dos projetos PEstOE/MAT/UI4080/2011, PEst-C/MAT/UI0144/ 2011 e PEst-C/MAT/UI4106/2011.

\section{REFERÊNCIAS}

Apple, M. L., Berger, R. D., Saul, J. P., Smith, J. M., \& Cohen, R. J. (1989). Beat to beat variability in cardiovascular variables: Noise or music? Journal of the American College of Cardiology, 14, 1139-1148. doi: 10.1016/0735-1097(89)9040 8-7

Baillie, R. T., Cecen, A. A., \& Erkal, C. (2009). Normal heartbeat series are nonchaotic, nonlinear, and multifractal: New evidence from semiparametric and parametric tests. Chaos, 19, 028503. doi: $10.1063 / 1.3152006$

Baumert, M., Javorka, M., Seeck, A., Faber, R., Sanders, P., \& Voss, A. (2012). Multiscale entropy and detrended fluctuation analysis of QT interval and heart rate variability during normal pregnancy. Computers in Biology and Medicine, 42(3), 347-352. doi: 10.1016/j.comp biomed.2011.03.019

Bigger, J. T., Steinman, R. C., Rolnitzky, L. M., Fleiss, J., Albrecht, P., \& Cohen, R. J. (1996). Power law behavior of RR-interval variability in healthy middle-aged persons, patients with recent acute myocardial infarction, and patients with heart transplants. Circulation, 93, 21422151. doi: 10.1161/01.CIR.93.12.2142

Boukhan, P., Oppenheim, G., \& Taqqu, M. (2003). Theory and Applications of Long-Range Dependence. Cambridge: Birkhäuser.

Brockwell, P. J., \& Davis, R. A. (1991). Time Series: Theory and Methods ( $\mathrm{a}^{\mathrm{a}}$ ed.). New York: Springer-Verlag.

Cerutti, S., Esposti, F., Ferrario, M., Sassi, R., \& Signorini, M. G. (2007). Long-term invariant parameters obtained from 24-h Holter recordings: A comparison between different analysis techniques. Chaos, 17(1), 0151089. doi: 10.1063/1.2437155 
Hurst, H. E. (1951). Long-term storage capacity of reservoirs. Transactions of the American Society of Civil Engineers, 116, 770-799.

Ivanov, P. C., Bunde A., Amaral L. A. N., Havlin S., Jritsch-Yelle J., Baevsky, R. M., ..., Goldberger A. L. (1999). Sleep-wake differences in scaling behavior of the human heartbeat: Analysis of terrestrial and long-term space flight data. Europhysics Letters, 48, 594-600. doi: 10.1209/ epl/i1999-00525-0

Iyengar, N., Peng, C. K., Morin, R., Goldberger, A. L., \& Lipsitz, L. A. (1996). Age-related alterations in the fractal scaling of cardiac interbeat interval dynamics. American Journal of Physiology, 271, R1078-1084.

Kobayashi, M., \& Musha, T. (1982). 1/f fluctuation of heartbeat period. IEEE Transactions on Biomedical Engineering, 29(6), 456-457. doi: 10.1109/TBME.1982.324972

Leite, A., Rocha, A., Silva, M., \& Costa, O. (2006). Modelling long-term heart rate variability: An ARFIMA approach. Biomedizinische Technik, 51, 215-219. doi: 10.1515/BMT.2006.040

Leite, A., Rocha, A. P., Silva, M. E., Gouveia, S., Carvalho, J., \& Costa, O. (2007). Long-range dependence in heart rate variability data: ARFIMA modelling vs detrended fluctuation analysis. Computers in Cardiology, 98(34), 21-24. doi: 10.1109/CIC.2007.4745411

Mäkikallio, T. H., Perkiömäki, J. S., \& Huikuri, H. V. (2004). Nonlinear dynamics of RR intervals. In M. M. Malik, \& A. J. Camm (Eds.), Dynamic Electrocardiography (pp. 22-30). Massachusetts: Blackwell Futura.

Malik, M., \& Camm, A. J. (1995). Heart rate variability. New York: Futura Publishing Company.

Peng, C.-K., Havlin, S., Stanley, H. E., \& Golberger, A. L (1995). Quantification of scaling exponents and crossover phenomena in nonsta- tionary heartbeat time series. Chaos, 5, 82-87. doi: 10.1063/1.166141

Penzel, T., Kantelhardt, J. W., Grote, L., Peter, J. H., \& Bunde, A. (2003). Comparison of detrended fluctuation analysis and spectral analysis for heart rate variability in sleep and sleep apnea. IEEE Transactions on Biomedical Engineering, 50, 1143-1151. doi: 10.1109/TBME.2003.817636

Pikkujämsä, S. M., Mäkikallio, T. H., Sourander, L. B., Räihä, I. J., Puukka P., Skyttä, J., ... Huikuri, H. V. (1999). Cardiac interbeat interval dynamics from childhood to senescence: comparison of conventional and new measures based on fractals and chaos theory. Circulation, 100, 393-399. doi: 10.1161/01.CIR.100.4.393

Saul, J. P., Albrecht, P., Berger, R. D., \& Cohen, R. J. (1987). Analysis of long term heart rate variability: Methods, 1/f scaling and implications. Computers in Cardiology, 14, 419-422.

Signorini, M. G., Sassi, R., \& Cerutti, S. (2001). Working on the NOLTISALIS Database: Measurement of nonlinear properties in heart rate variability signals. In Proceedings of IEEE-EMBS International Conference, Istanbul, Turkey.

Struzik, Z. R., Hayano, J., Soma, R., Kwak, S., \& Yamamoto, Y. (2006). Aging of complex heart rate dynamics. IEEE Transactions on Biomedical Engineering, 53(1), 89-94. doi: 10.1109/TBME. 2005.859801.

Task Force of European Society of Cardiology and the North American Society of Pacing Electrophysiology (1996). Heart rate variability: standards of measurement, physiological interpretation and clinical use. Circulation, 93, 10431065. doi: 10.1161/01.CIR.93.5.1043

Yamamoto, Y., \& Hughson, R. L. (1991). Coarsegraining spectral analysis: New method for studying heart rate variability. Journal of Applied Physiology, 71, 1143-1150.

(c) EY-No Todo o conteúdo da revista Motricidade está licenciado sob a Creative Commons, exceto quando especificado em contrário e nos conteúdos retirados de outras fontes bibliográficas. 